\title{
Celecoxib Exerts a Therapeutic Effect Against Demyelination by Improving the Immune and Inflammatory Microenvironments
}

This article was published in the following Dove Press journal: Journal of Inflammation Research

\section{Peipei Cao' \\ Hao Zhang $\mathbb{( D}^{2}$ \\ Huiling Meng' \\ Yajia Cheng' \\ Haiqi $X u^{3}$ \\ Siwen Zang' \\ Zongjin Li (D) \\ Jianlin Cui ${ }^{1,4}$ \\ Yuhao $\mathrm{Li} \mathbb{1}^{1,5}$}

'Nankai University School of Medicine, Tianjin, People's Republic of China; ${ }^{2}$ Department of Neurosurgery, Tianjin Medical University General Hospital, Tianjin, People's Republic of China; ${ }^{3}$ Faculty of Life Science, University of Liverpool, Liverpool, UK; ${ }^{4}$ Medical International Collaborative Innovation Center, Nankai University School of Medicine, Tianjin, People's Republic of China; ${ }^{5}$ Department of Pathology, Nankai University School of Medicine, Tianjin, People's Republic of China
Background: The myelin sheath can be damaged by genetic and/or environmental factors, leading to demyelinating diseases, for which effective treatments are lacking. Recently, cyclooxygenase-2 (COX-2) overexpression was detected in demyelinating lesions both in patients and animal models, opening an avenue for promoting endogenous remyelination. The aim of this study was to investigate the therapeutic effect of celecoxib, a selective COX2 inhibitor, against demyelination in a zebrafish model.

Methods: The biotoxicity of celecoxib was evaluated on zebrafish embryos. Metronidazole was used to deplete the oligodendrocytes in Tg (mbp:nfsB-egfp) transgenic fish. Celecoxib was then administered both in larvae and adults. The regeneration of the myelin sheath and the underlying mechanisms were explored by immunohistochemistry, flow cytometry, Western blot analysis, quantitative real-time polymerase chain reaction, and behavioral test. Results: Celecoxib had low in vivo toxicity. A stable and practical demyelination model was established by metronidazole induction. Following celecoxib treatment, the number of oligodendrocytes was increased significantly and the concentric structure of the myelin sheath reappeared. The locomotor ability was notably improved and was close to its physiological levels. The expression of arg1, mrc1, il-10, and il-4 was upregulated, while that of il-1 $\beta$, il-12, tnf- $\alpha$, il-6, caspase- 3 and caspase-7 was downregulated.

Conclusion: Inhibition of COX-2 contributed to the transformation of microglia/macrophages from the M1 to the M2 phenotype, improved the inflammatory microenvironment, and suppressed caspase-dependent apoptosis, thus exerting a therapeutic effect against demyelination.

Keywords: celecoxib, demyelination, oligodendrocytes, remyelination, zebrafish

\section{Introduction}

The myelin sheath is a multilayered membranous structure that protects, supports, and nourishes axons, and increases the conduction velocity of nerve impulses. ${ }^{1}$ Concentric myelin membranes originate from oligodendrocytes in the central nervous system (CNS) and Schwann cells in the peripheral nervous system. ${ }^{2,3}$ Internal or external conditions may damage the myelin sheath, leading to demyelination. Multiple sclerosis (MS) is the most common chronic neuroinflammatory demyelinating disease, and it mainly occurs in young people, especially women. ${ }^{4}$ The hallmark of MS is the formation of sclerosing plaques in the brain, spinal cord, optic nerve, etc., due to the depletion of oligodendrocytes accompanied by axonal degeneration and inflammatory cell infiltration. At the early stage, the myelin
Department of Pathology, Nankai

University School of Medicine, 94 Weijin

Road, Tianjin 30007I, People's Republic of

China

Tel +8622 23502554

Email liyuhao@nankai.edu.cn

Jianlin Cui

Medical International Collaborative Innovation Center, Nankai University

School of Medicine, 94 Weijin Road,

Tianjin 30007I, People's Republic of

China

Tel +8622 23502554

Email cuijianlin@nankai.edu.cn
Journal of Inflammation Research 2020:13 1043-1055

1043

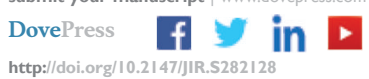


sheath can be regenerated to a certain degree. Clinical studies demonstrated that individuals with more remyelination capacity had milder clinical disability symptoms. ${ }^{5,6}$ However, this regeneration is not long-lasting. ${ }^{7}$ Hence, improving endogenous myelin regeneration is a useful approach for the treatment of MS.

Cyclooxygenase (COX) is a rate-limiting enzyme in arachidonic acid metabolism. There are two isoforms of COX, namely, COX-1 and COX-2. COX-1, the constitutive isoform, is highly expressed in normal tissues. In contrast, the COX-2 inducible isozyme is expressed at much lower levels under physiological conditions. High expression of COX-2 is found in sites of inflammation, tissue damage, and malignant transformation. ${ }^{8}$ Recently, it was reported that COX-2 overexpression can be detected at active demyelinating lesions in MS patients, as well as in animal models of demyelination. ${ }^{9-11}$ Celecoxib, a COX2 inhibitor, is a nonsteroidal anti-inflammatory drug (NSAID) and widely used to relieve the symptoms of arthritis and pain. Its benzenesulfonamide structure had high selectivity for the COX-2 receptor, leading to a decrease in the synthesis of prostaglandin $\mathrm{E}_{2}$. However, celecoxib did not inhibit COX-1. Celecoxib was also reported to treat tumors, cardiovascular diseases, retinopathy, fatty liver, etc. To our knowledge, its effects on demyelination are still not understood.

Zebrafish (Danio rerio), a member of the teleost family, has become an ideal animal model for investigating neurodegenerative diseases. ${ }^{12}$ The structure of the myelin sheath and myelination process are very similar between zebrafish and mammals. ${ }^{13}$ In zebrafish, a noncompact myelin structure appears at 4 days post fertilization (dpf), while a tight myelin sheath forms at $7 \mathrm{dpf}$. Thus, myelination occurs within the first week, which greatly limits the time. The mechanisms of myelination can be explored or drugs for remyelination can be screened in a short period. ${ }^{14}$ Since the myelin basic protein (mbp) gene is a specific marker of the myelin sheath and plays a critical role during demyelination, we generated a stable and heritable transgenic zebrafish line in our previous work. In Tg (mbp:nfsB-egfp) fish, oligodendrocytes continuously express a fusion protein consisting of enhanced green fluorescent protein (GFP) and nitroreductase (NTR) from the mbp promoter. ${ }^{15}$ Therefore, oligodendrocytes can be specifically ablated by treatment with metronidazole (Mtz). This model not only allows demyelination to be visualized in vivo but also offers a feasible tool for evaluating the response of drugs to remyelination.
In this study, we induced demyelination in Tg (mbp: nfsB-egfp) zebrafish and investigated the therapeutic effects of celecoxib. We observed the following: (1) the in vivo toxicity of celecoxib on zebrafish embryos; (2) the process of remyelination and its functional changes following celecoxib treatment; and (3) the underlying mechanisms by which celecoxib governs remyelination. Our study might help develop a celecoxib-based treatment approach for demyelinating diseases.

\section{Materials and Methods}

\section{Experimental Animals}

Tg (mbp:nfsB-egfp) transgenic zebrafish and wild-type zebrafish (AB strain) were used in this study. Fish were raised at $28.5^{\circ} \mathrm{C}$ on a $14 / 10$-hour light/dark cycle. ${ }^{16}$ Embryos were incubated in $\mathrm{E} 3$ medium $(5 \mathrm{mmol} / \mathrm{L} \mathrm{NaCl}$, $0.17 \mathrm{mmol} / \mathrm{L} \mathrm{KCl}, 0.33 \mathrm{mmol} / \mathrm{L} \mathrm{CaCl}_{2}$, and $0.33 \mathrm{mmol} / \mathrm{L}$ $\mathrm{MgSO}_{4}, \mathrm{pH}$ 7.2) and staged by hours post fertilization (hpf) or days post fertilization (dpf). All zebrafish experimental protocols were approved by the Institutional Animal Care and Use Committee of Nankai University and complied with guide for the care and use of laboratory animals by National Institutes of Health (NIH).

\section{In vivo Toxicity Analysis}

To test the toxicity of celecoxib, embryos were collected at $1 \mathrm{hpf}$, placed in a 6-well plate (10 embryos/well), and exposed to celecoxib (S1261; Selleck Chemicals, Houston, TX, USA) at concentrations of $0.5,1,2$, and 4 $\mu \mathrm{M}$. In the control group, the same number of embryos was cultured in E3 medium. Embryonic phenotypes were imaged with a DP72 digital camera mounted on an SZX16 stereomicroscope (Olympus Corporation, Tokyo, Japan) at 6, 24, 48, and $72 \mathrm{hpf}$. In addition, the survival rate and hatching rate were calculated to evaluate the in vivo toxicity.

\section{Metronidazole Induction and Celecoxib}

\section{Treatment}

In this study, metronidazole (Mtz; M3761, Sigma, St. Louis, MO, USA) was used to induce demyelination. ${ }^{15,17}$ Briefly, $20 \mathrm{Tg}$ (mbp:nfsB-egfp) adults and 100 larvae were continuously induced with $5 \mathrm{mM}$ Mtz-0.2\% dimethyl sulfoxide (DMSO; V900090, Sigma)system water or E3 medium in the dark for 4 days. The same number of adults and larvae were incubated in $0.2 \%$ DMSO-system water or E3 medium as controls. Celecoxib 
was administered following Mtz induction. Celecoxib was dissolved in DMSO to prepare a stock solution at a concentration of $200 \mathrm{mM}$. Adults and larvae were then treated with celecoxib at different concentrations for 6 and 3 days, respectively. The control adults and larvae were incubated with $0.2 \%$ DMSO for 6 and 3 days, respectively. Both the Mtz induction experiments and the celecoxib treatment experiments were repeated 3 times.

\section{Quantitative Real-Time Polymerase Chain Reaction (qRT-PCR)}

Total RNA was extracted from larvae and adult brain tissues using TRIzol reagent (Thermo, Austin, TX, USA) according to the manufacturer's protocol. Reverse transcription (RT) was carried out using TransScript FirstStrand cDNA Synthesis SuperMix (TransGen Biotech, Beijing, China). Real-time PCR was performed using TransStart Top Green qPCR SuperMix (TransGen). The protocol was as follows: 30 seconds at $94^{\circ} \mathrm{C}$ followed by 45 cycles of 5 seconds at $94^{\circ} \mathrm{C}, 30$ seconds at $60^{\circ} \mathrm{C}$, and 10 seconds at $72^{\circ} \mathrm{C}$. The relative expression of mRNA was calculated by the $2^{-\Delta \Delta \mathrm{Ct}}$ method. The experiment was repeated 3 times for each gene. The gene-specific primer sequences are listed in Supplementary Table S1.

\section{Immunohistochemistry}

Adult zebrafish were anesthetized with 0.1\% 3-aminobenzoic acid ethyl ester methanesulfonate (MS-222; A5040, Sigma) and euthanized immediately. The brain, optic nerve, and spinal cord were dissected, fixed in $4 \%$ paraformaldehyde (PFA) overnight, dehydrated in $20 \%$ sucrose in $0.1 \mathrm{M}$ phosphatebuffered saline (PBS, $\mathrm{pH} 7.4$ ) at room temperature, embedded in optimal cutting temperature compound (Sakura Finetek, Torrance, CA, USA) and processed for cryosectioning at 8 $\mu \mathrm{m}$. Immunohistochemistry was performed as previously described. ${ }^{18}$ The primary antibodies were an anti-GFP antibody (1:500; ab6556, Abcam, Cambridge, MA, USA), which was used to label oligodendrocytes in Tg (mbp:nfsB-egfp) zebrafish, and an anti-COX-2 antibody (1:200; 55070-1-1AP, Proteintech, Chicago, IL, USA). The secondary antibody was a FITC-conjugated antibody (1:500; Millipore, Billerica, MA, USA). 4'6-Diamidino-2-phenylindole (DAPI) was used to counterstain the nuclei.

\section{Western Blot Analysis}

Brain tissues from adult zebrafish and larvae were collected and lysed in RIPA buffer containing protease inhibitors. The protein concentrations were measured using the BCA Protein Assay Kit (CW0014S; CWBiotech, Beijing, China). Western blotting was performed as previously described. ${ }^{19}$ Two antibodies, namely, an anti-MBP antibody (1:500; \#55,811, Anaspec, Fermont, CA, USA) and an anti-COX-2 antibody (1:1000; Proteintech), were used in this study.

\section{Flow Cytometry}

Following Mtz induction, adult fish were divided into two groups: the celecoxib group, in which fish were treated with celecoxib, and the control group, in which fish were cultured with E3 medium. The adult fish were anesthetized with $0.1 \%$ MS-222 and immediately euthanized. To prepare a single cell suspension, 3 brain tissues from each group were dissected, washed twice in prechilled 0.1 M PBS, dissociated in $300 \mu \mathrm{L}$ of $0.25 \%$ trypsin solution, and suspended in 0.1 M PBS. Brain tissues from wild-type fish were used to remove autofluorescence interference. Then, GFP-positive cells from the two groups were sorted with a FACS Aria III system (Becton Dickinson, Franklin Lakes, NJ, USA) with a Coherent Innova 70 laser at 488 $\mathrm{nm}$ at $4^{\circ} \mathrm{C}$. The above experiment was repeated three times.

\section{Behavioral Analysis}

Tg (mbp:nfsB-egfp) larvae were subjected to behavioral tests. Larvae treated with Mtz and celecoxib were used as the $\mathrm{Mtz}^{+} /$celecoxib ${ }^{+}$group. Larvae induced only with Mtz were used as the $\mathrm{Mtz}^{+} /$celecoxib ${ }^{-}$group. Larvae not treated with any agent were used as the control group. Six larvae from each group were collected, washed in E3 medium, and placed in a 24-well plate. Each well contained $2 \mathrm{~mL}$ of E3 medium and one larva. Following 15 minutes of aquarium acclimation, locomotion was tracked with a camera positioned above the plate for 10 minutes. All digital tracks and heat maps were analyzed by Ethovision XT software (Noldus Information Technology, Wageningen, the Netherlands). Four parameters, including total distance, average speed, total movement time, and maximum acceleration, were analyzed. The behavioral test was repeated three times.

\section{Statistical Analysis}

Statistical analysis was performed using Prism software (version 7.0, GraphPad Software, La Jolla, USA). The values are shown as the mean \pm standard deviation (SD) or standard error of the mean (SEM). $P<0.05$ was 
considered statistically significant. Values from two groups were compared with Student's $t$-test. Data from more than two groups were analyzed using one-way analysis of variance (ANOVA).

\section{Results}

\section{Celecoxib Has Low in vivo Toxicity}

To evaluate the potential toxicity of celecoxib, zebrafish embryos at 1 hour post fertilization (hpf) were incubated with $0.5,1,2$, or $4 \mu \mathrm{M}$ celecoxib. As shown in Figure 1A, no obvious malformation was observed in the celecoxibexposed groups at any time point $(6,24,48$, or $72 \mathrm{hpf})$. We then calculated the survival rate at 6,12 , and $24 \mathrm{hpf}$ and the hatching rate at 48,60 , and 72 hpf. No significant difference was found between the groups (Figure $1 \mathrm{~B}$ and $\mathrm{C}$; ANOVA, $P>0.05$ ). These data indicate that celecoxib has low vivo toxicity and does not affect the development of zebrafish embryos.

\section{Mtz Specifically Depletes Oligodendrocytes in Tg (mbp:nfsB-Egfp) Zebrafish}

The Mbp gene is expressed specifically in differentiated oligodendrocyte lineage cells and is used as a marker of the myelin sheath. ${ }^{20-22}$ In this study, the demyelination process was established in $\mathrm{Tg}$ (mbp:nfsB-egfp) adults and larvae. We evaluated the expression of GFP by immunohistochemical analysis of cryosections from adults following 4 days of Mtz induction. Fewer GFP-expressing cells were detected in the brains (Figure 2A), optic nerves (Figure 2C), and spinal cords (Figure 2E) of adults in the Mtz-induced group than those in the control group. In the Mtz-induced group, the myelin sheath of the spinal cord became incomplete and lost its concentric circular structure surrounding axons (Figure 2E). To quantify the changes, we analyzed the ratio of the number of GFPpositive cells to the number of nuclei (GFP/DAPI). The ratios in the brain, optic nerve, and spinal cord in the Mtzinduced group were lower than those in the control group (Figure 2B, D and F; Student's $t$-test, ${ }^{*} P<0.05,{ }^{* *} P<0.01$, $* * * P<0.001)$. Similarly, the Western blotting and qRTPCR results showed that the expression of MBP protein and mbp mRNA was remarkably decreased after Mtz induction (Figure $2 \mathrm{G}$ and $\mathrm{H}$; Student's $t$-test, *** $P<0.001)$. We also examined oligodendrocyte ablation in larvae. Following 4 days of induction, the fluorescence signals in the spinal cord in the Mtz-induced group were lower and more discontinuous than those in the control group (Supplementary Figure S1A). qRT-PCR and Western blot analysis indicated that Mtz induction resulted in a significant downregulation of $\mathrm{mbp}$ expression (Supplementary Figure S1B and $\underline{\mathrm{C}}$ ). The above data demonstrate that induction of Tg (mbp:nfsB-egfp) adults and larvae with Mtz leads to specific depletion of oligodendrocytes, and is thus an effective model of demyelination.

\section{Celecoxib Treatment Promotes}

\section{Remyelination in Demyelinated Zebrafish}

First, we tested the optimal concentration and duration of celecoxib treatment based on mbp expression. Following 4 days of Mtz induction, Tg (mbp:nfsB-egfp) adults were soaked in $0.5,1$, or $2 \mu \mathrm{M}$ celecoxib for 6 days, and mbp expression was significantly increased in a dose-dependent manner in the 1 and $2 \mu \mathrm{M}$-treated groups compared to the 0 and $0.5 \mu \mathrm{M}$-treated group (Supplementary Figure S2A; ANOVA, $\left.{ }^{* *} P<0.01\right)$. We then treated demyelinated adults with $2 \mu \mathrm{M}$ celecoxib for 2,4 , or 6 days. Compared with that in the untreated group, mbp expression was distinctly increased at 6 days post treatment (dpt; Supplementary Figure S2B; ANOVA, $* * P<0.01$ ). Therefore, we chose 2 $\mu \mathrm{M}$ and 6 days as the concentration and duration for celecoxib treatment in adults (Figure 3A). The effects of celecoxib treatment were verified by two approaches. First, we measured the number of GFP-positive cells in brain tissue by cell sorting since oligodendrocytes were specifically labeled with GFP. As shown in Figure 3B and $\mathrm{C}$, celecoxib treatment led to an increase in the number of oligodendrocytes (Student's $t$-test, $\quad * * P<0.01$ ). Furthermore, we examined the expression of GFP in the brain, spinal cord and optic nerve by immunohistochemistry and quantified the results based on the GFP/DAPI ratio (Figure 3D-I). To evaluate the effect of celecoxib treatment, three groups were set: control group (adults not treated with any agent), $\mathrm{Mtz}^{+} / \mathrm{celecoxib}^{-}$group (adults induced only with $\mathrm{Mtz}$ ), and $\mathrm{Mtz}^{+} /$celecoxib $^{+}$group (adults induced with Mtz and treated with celecoxib). The number of GFP-positive cells in the white matter of the brain was increased in the $\mathrm{Mtz}^{+} /$celecoxib $^{+}$group compared to the $\mathrm{Mtz}^{+} /$celecoxib ${ }^{-}$group (Figure 3D and E; ANOVA, $* * P<0.01)$. In the spinal cord, more GFPexpressing cells were detected in the $\mathrm{Mtz}^{+} /$celecoxib $^{+}$ group than in the $\mathrm{Mtz}^{+} /$celecoxib $^{-}$group, mainly on the ventral side, and the complete concentric structure of the 
A
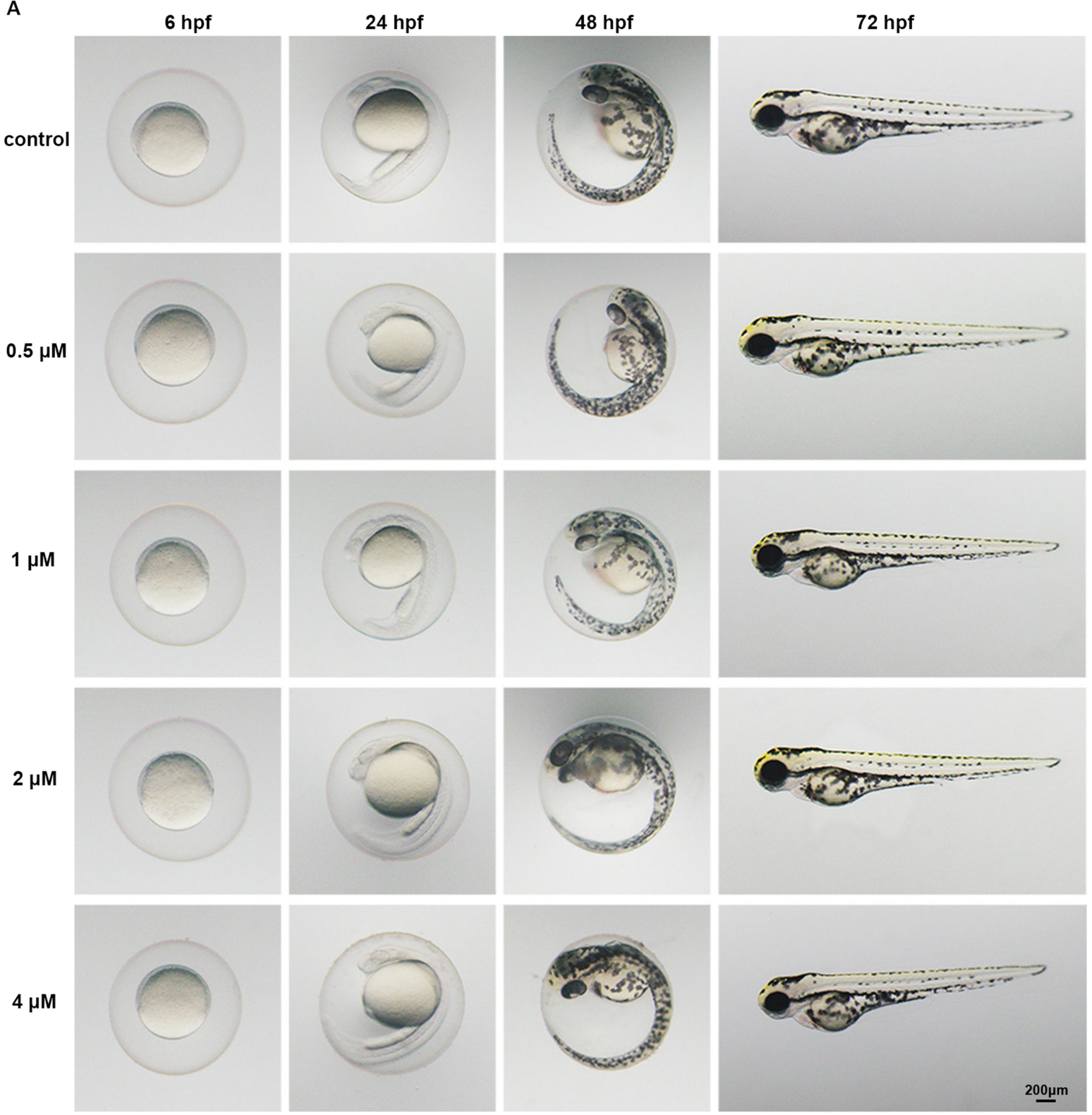

B

C
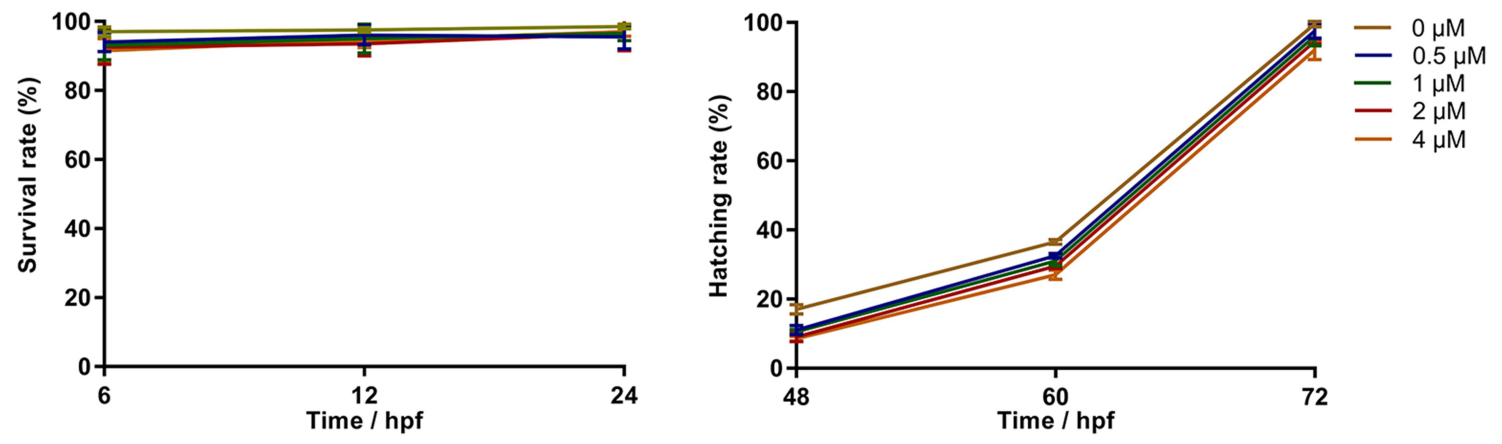

Figure I Phenotypes, survival rates and hatching rates of zebrafish embryos following celecoxib exposure. (A) Phenotypes of embryos from 6 to 72 hpf. (B and C) Statistical analysis of survival rate and hatching rate (ANOVA). Abbreviation: hpf, hours post fertilization. 
A

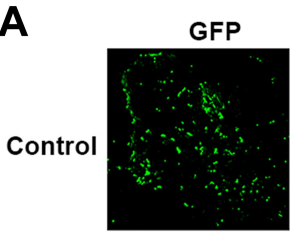

Mtz

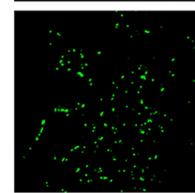

C

Control

Mtz
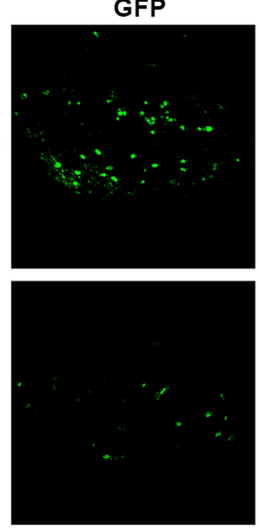

E

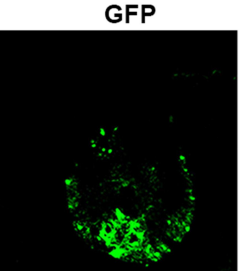

Mtz

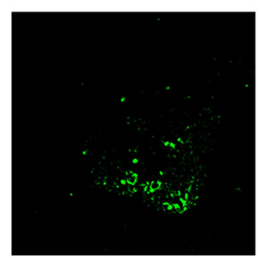

G

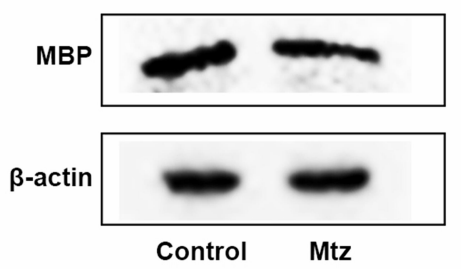

B

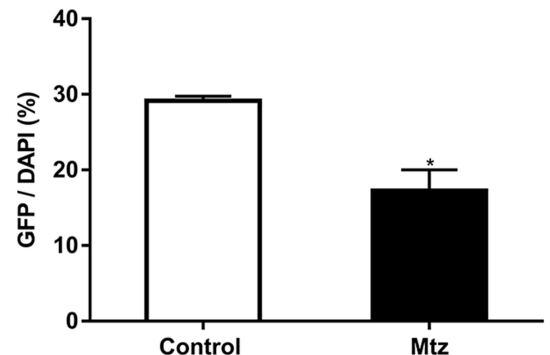

D

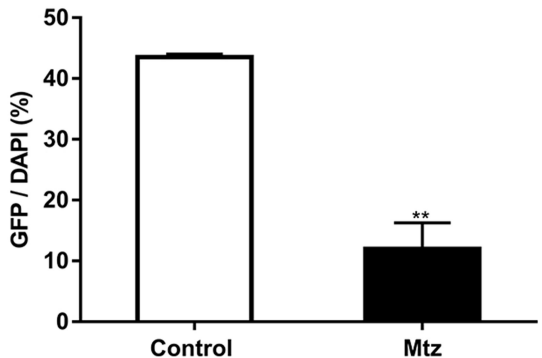

F
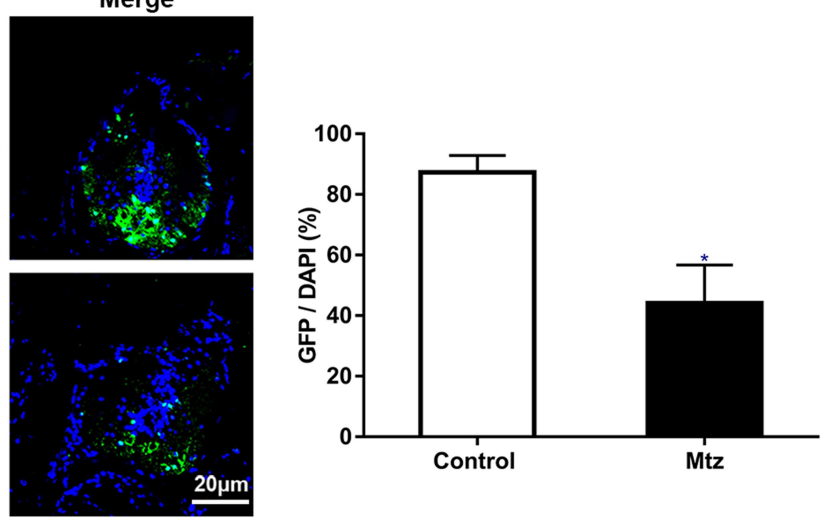

H

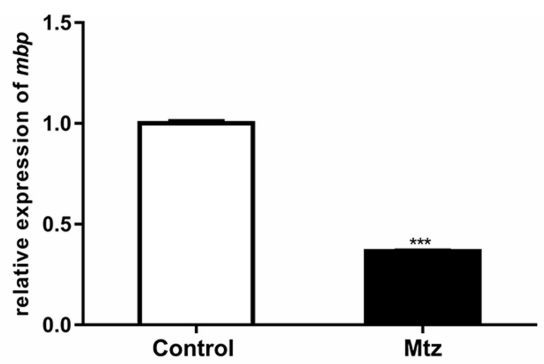

Figure $2 \mathrm{Mtz}$ induction leads to specific ablation of oligodendrocytes in adult Tg (mbp:nfsB-egfp) zebrafish. (A, C and E) Immunohistochemical staining of GFP in transverse sections of the brain (A), optic nerve (C) and spinal cord (E) in the control and Mtz-induced groups. Note that the ratio of GFP to DAPI was significantly decreased in the Mtz-induced group compared to the control group in the brain (B), optic nerve (D) and spinal cord (F) (Student's $t$-test). (G) The expression of MBP protein, as analyzed by Western blotting. (H) The relative expression of mbp mRNA, as analyzed by qRT-PCR. Note that the expression level in the Mtz-induced group was significantly decreased compared to that in the control group (Student's $t$-test). $* P<0.05 ; * * P<0.01 ; * * * P<0.00$ I. 
A

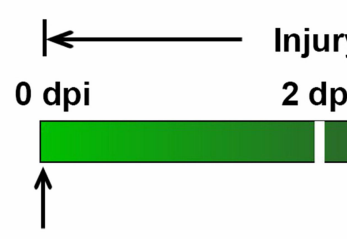

Mtz induction

B

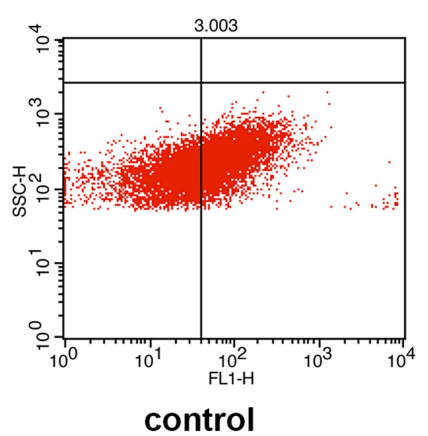

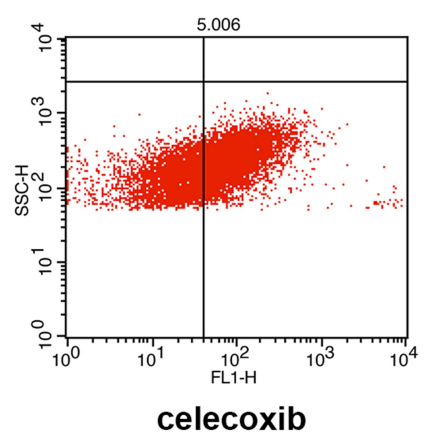

celecoxib
C

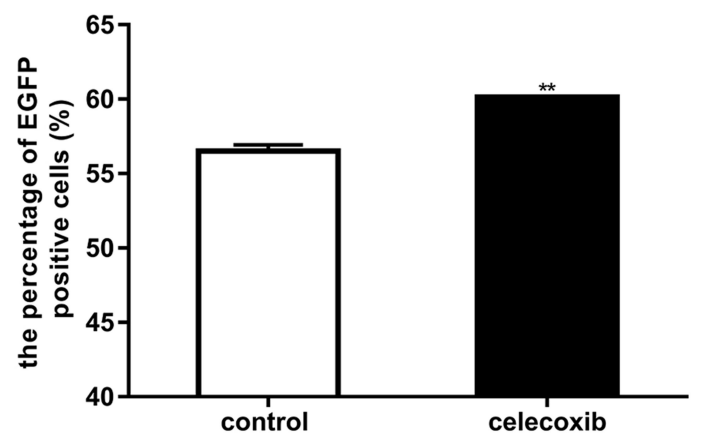

D

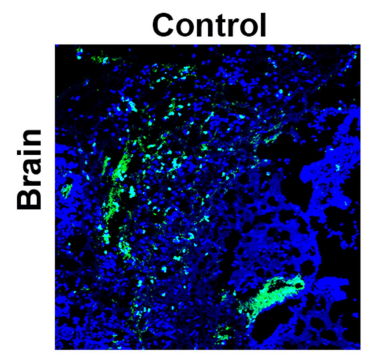

F

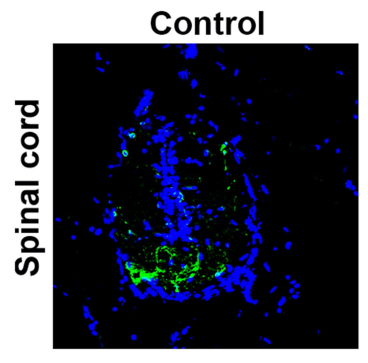

H

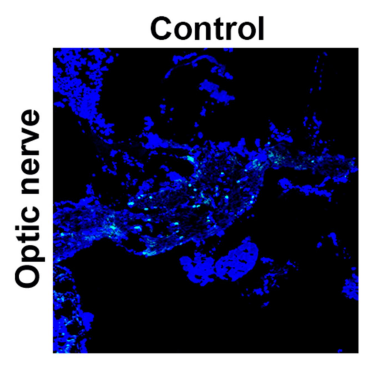

$\mathrm{Mtz}^{+} /$Celecoxib

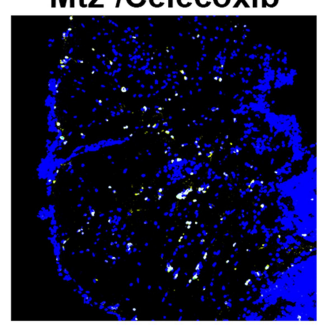

$\mathrm{Mtz}^{+} /$Celecoxib
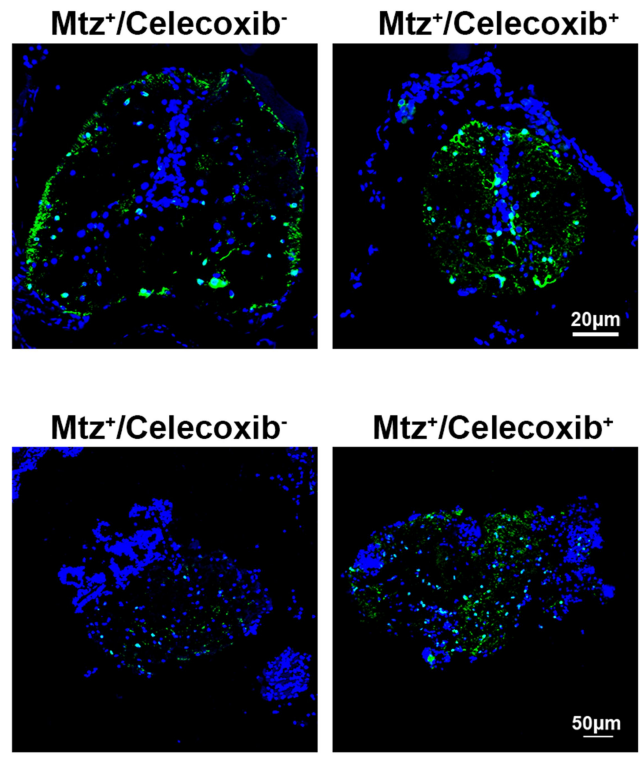

$\mathrm{Mtz}^{+} /$Celecoxib ${ }^{+}$
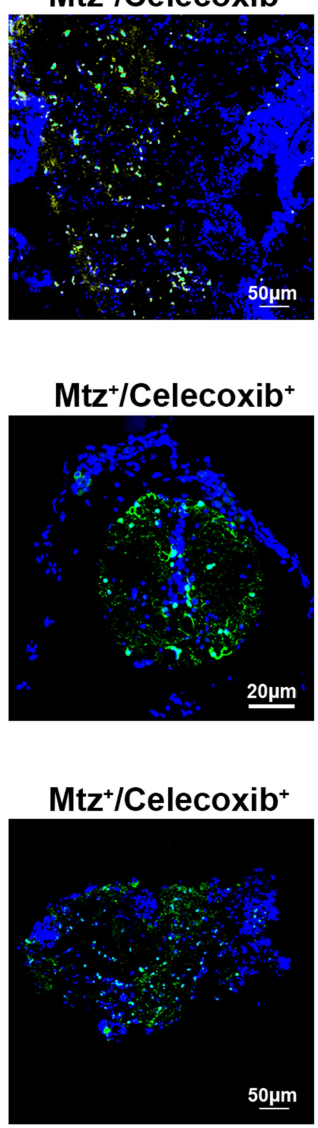

E

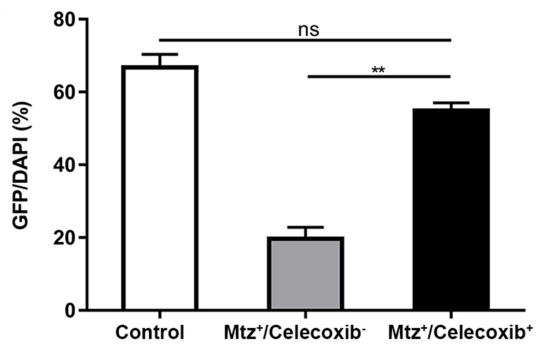

G

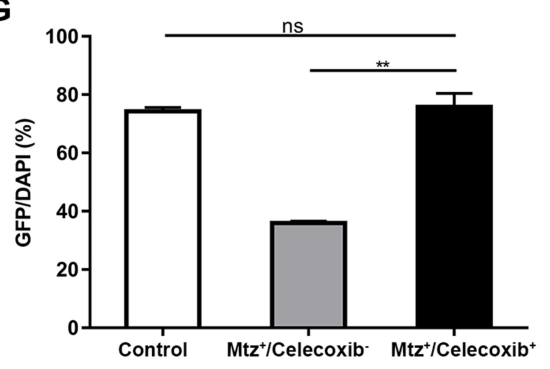

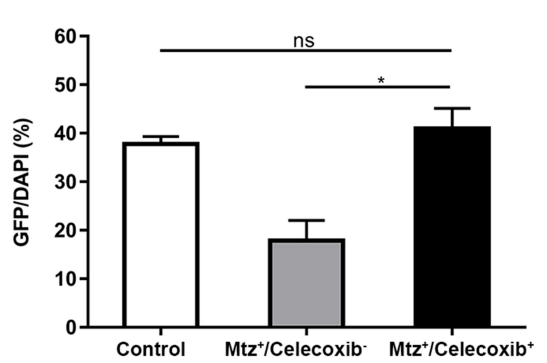

Figure 3 Cell sorting of oligodendrocytes and GFP-expressing cells in adult Tg (mbp:nfsB-egfp) zebrafish following celecoxib treatment. (A) Timeline of Mtz induction and celecoxib treatment. (B) GFP-positive cell sorting by flow cytometry in the control and celecoxib-treated groups. Note the increased number of GFP-positive cells in the celecoxib-treated group compared to the control group (C; Student's $t$-test). (D, F and $\mathbf{H}$ ) Immunostaining of GFP expression on transverse sections taken through brain (D), spinal cord (F) and optic nerve $(\mathbf{H})$ in control, $\mathrm{Mtz}^{+} /$celecoxib $^{-}$group, and $\mathrm{Mtz}^{+} /$celecoxib $^{+}$groups. Note that the ratios of GFP/DAPI increase significantly in Mtz ${ }^{+}$ celecoxib $^{+}$group (E, G and I; ANOVA). $* P<0.05 ; * P<0.0$ I.

Abbreviation: ns, not significant. 
myelin sheath surrounding axons was relatively restored (Figure 3F and G; ANOVA, $* * P<0.01$ ). Similarly, in the optic nerve, more positive cells were observed in a linear pattern that matched its physiological distribution in the $\mathrm{Mtz}^{+} /$celecoxib $^{+}$group (see; ${ }^{23}$ Figure $3 \mathrm{H}$ and I; ANOVA, $\left.{ }^{*} P<0.05\right)$. Surprisingly, the expression pattern of GFP in the brain, spinal cord, and optic nerve from $\mathrm{Mtz}^{+}$/ celecoxib $^{+}$group was close to the control group (Figure $3 \mathrm{D}, \mathrm{F}$ and $\mathrm{H}$ ) and no significant difference was found in GFP/DAPI ratio between two groups (Figure 3E, G and I; ANOVA, $P>0.05$ ).

We also evaluated the remyelination process in larvae. Following 4 days of Mtz induction, larvae were incubated with $2 \mu \mathrm{M}$ celecoxib for 3 days (Figure 4A). GFP signals in the spinal cord were recorded on each dpt. At $1 \mathrm{dpt}$, there was little difference between the control and celecoxibtreated groups. However, in the celecoxib-treated group, the GFP signals were slightly higher than those in the control larvae at $2 \mathrm{dpt}$ and were much brighter than those in control larvae at $3 \mathrm{dpt}$ (Figure 4B). We further examined mbp expression. In the celecoxib-treated group, MBP protein expression increased steadily with treatment time, while little change was found in the control group (Figure 4C). The qRT-PCR results showed that the expression of mbp mRNA was significantly upregulated following celecoxib treatment (Figure 4D; Student's $t$-test, ${ }^{* *} P<0.01$, *** $P<0.001)$. Taken together, the above data indicate that celecoxib treatment increases the number of oligodendrocytes and mbp expression and restores myelin structure, thus promoting remyelination in demyelinated zebrafish.

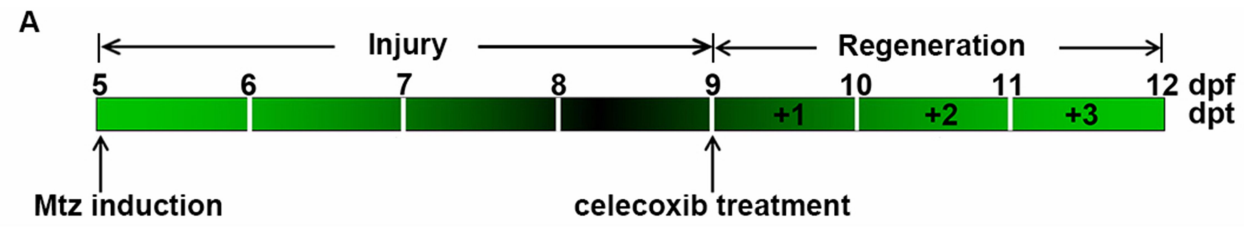

B
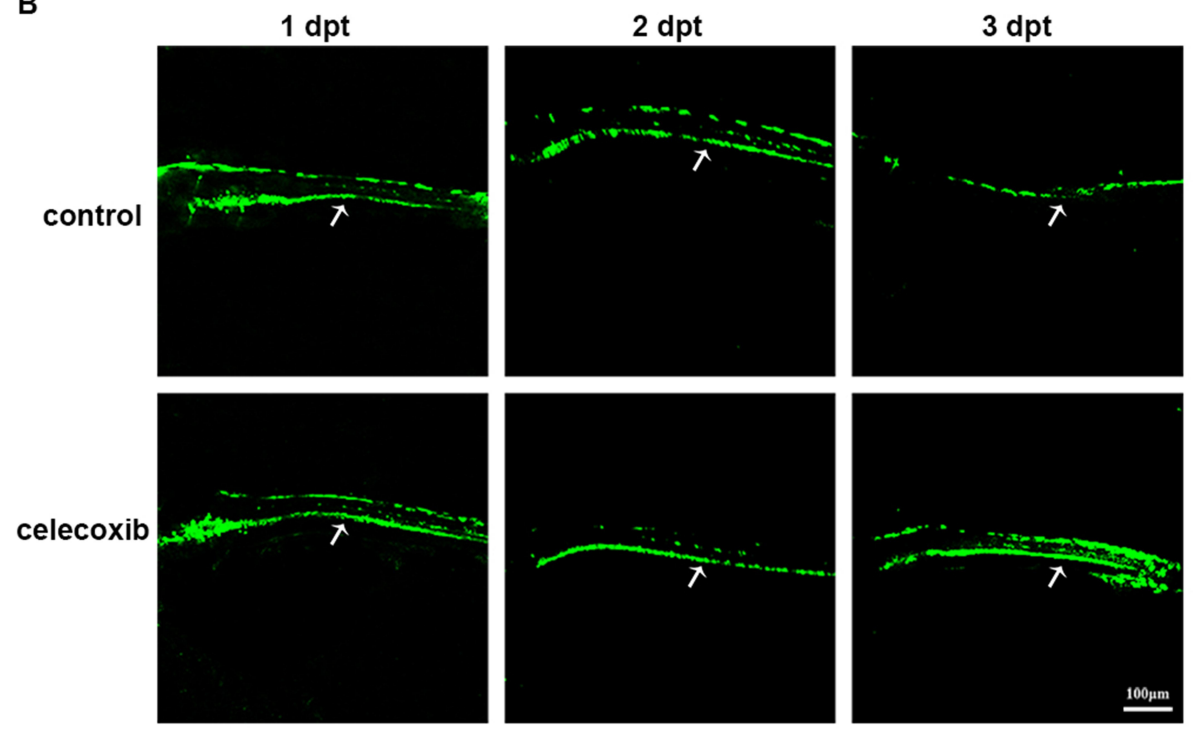

C

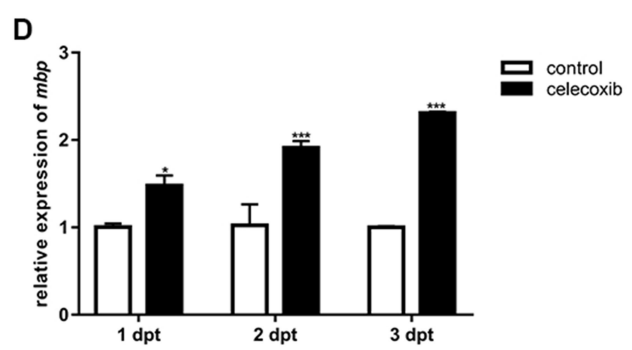

Figure 4 Celecoxib treatment promotes remyelination in Tg (mbp:nfsB-egfp) larvae. (A) Timeline of Mtz induction and celecoxib treatment in larvae. (B) Images of GFP fluorescence in the spinal cord (arrows) of demyelinated (control) and celecoxib-treated larvae at I, 2 and 3 days post treatment (dpt). (C) The expression of MBP protein, as analyzed by Western blotting, at I, 2, and $3 \mathrm{dpt}$ in the control and celecoxib-treated groups. (D) Relative mbp mRNA expression in the control and celecoxib-treated groups (Student's $t$-test). Abbreviations: dpf, days post fertilization; dpt, days post treatment. $* * p<0.01$; $* * * p<0.001$. 


\section{Celecoxib Treatment Restores the Locomotor Abilities of $\mathrm{Tg}$ (mbp: nfsB-Egfp) Larvae}

To evaluate the functional changes following celecoxib treatment, we subjected larvae to a behavioral test. Figure 5A shows the digital tracks and corresponding heat maps. Following celecoxib treatment, the total distance, movement time, velocity, and maximum acceleration of the $\mathrm{Mtz}^{+} /$celecoxib $^{+}$group were significantly higher than those of the $\mathrm{Mtz}^{+} /$celecoxib ${ }^{-}$group (Figure 5B; ANOVA, $* P<0.05, \quad * * * P<0.001)$. Moreover, no obvious difference was observed between the control group and $\mathrm{Mtz}^{+} /$celecoxib $^{+}$group (Figure 5B). These results suggest that celecoxib treatment remarkably improves locomotor ability, restoring it to almost the normal level.

\section{Celecoxib Treatment Improves the Immune and Inflammatory Microenvironments and Suppresses Apoptosis}

We investigated the underlying mechanisms of celecoxib treatment on four parameters in adult zebrafish. First, we tested the expression of COX-2 following celecoxib treatment. Immunostaining for COX-2 was performed on brain tissue sections (Figure 6A). The results showed that fewer
COX-2-expressing cells were detected in the celecoxibtreated group than the control group. Western blot analysis showed decreased COX-2 expression (Figure 6B). Cox-2 mRNA expression was also downregulated in the celecoxib-treated group compared to the control group (Figure 6C; Student's $t$-test, $* P<0.05$ ). These data demonstrate that celecoxib inhibits the expression of COX-2. Second, we explored the correlation between microglial/ macrophage polarization phenotype and celecoxib treatment. The expression of M1 type markers (il-1 $\beta$ and il12) was decreased in the celecoxib group compared to the control group. In contrast, the expression of M2 phenotype markers (arg1 and mrc1) was increased (Figure 6D; Student's $t$-test, $\quad * P \quad<0.05, \quad * * P<0.01, \quad * * * P<0.001)$. These data reveal that celecoxib treatment contributes to the transformation of microglia/macrophages from the M1 to the M2 phenotype in the CNS. Third, we evaluated the inflammatory response by qRT-PCR. The expression of anti-inflammatory cytokines (il-10 and il-4) was upregulated, while that of two pro-inflammatory cytokines ( $\operatorname{tnf}-\alpha$ and il-6) were decreased in the celecoxib-treated group compared to the control group (Figure 6E; Student's $t$-test, $* * P<0.01, * * * P<0.001)$. Finally, we determined that the expression of two apoptotic factors, namely, caspase- 3 and caspase-7, was decreased in the celecoxibtreated group compared to the control group (Figure 6F; Student's $t$-test, $\left.{ }^{*} P<0.05\right)$. To verify the above findings, we also tested the expression of COX-2, M1/M2 markers,

A

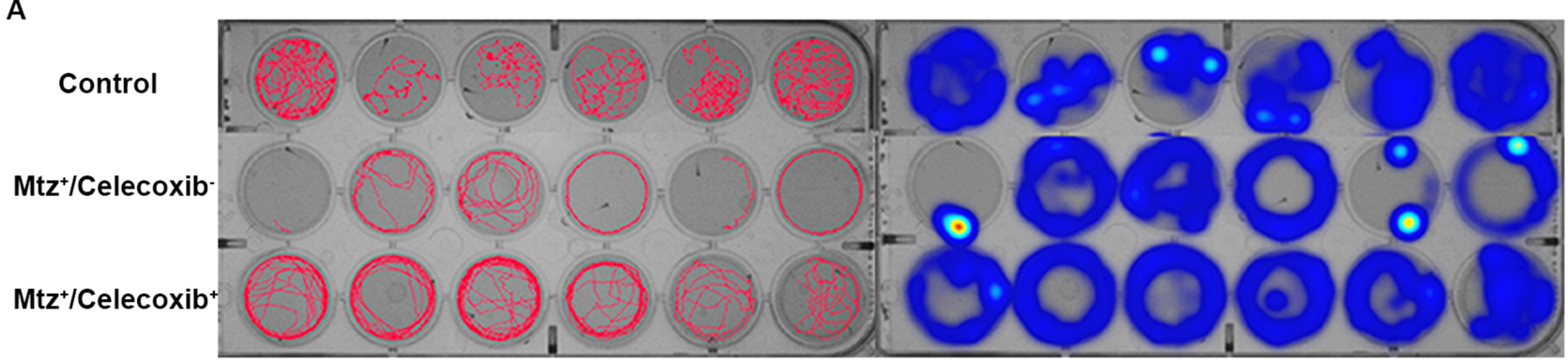

B
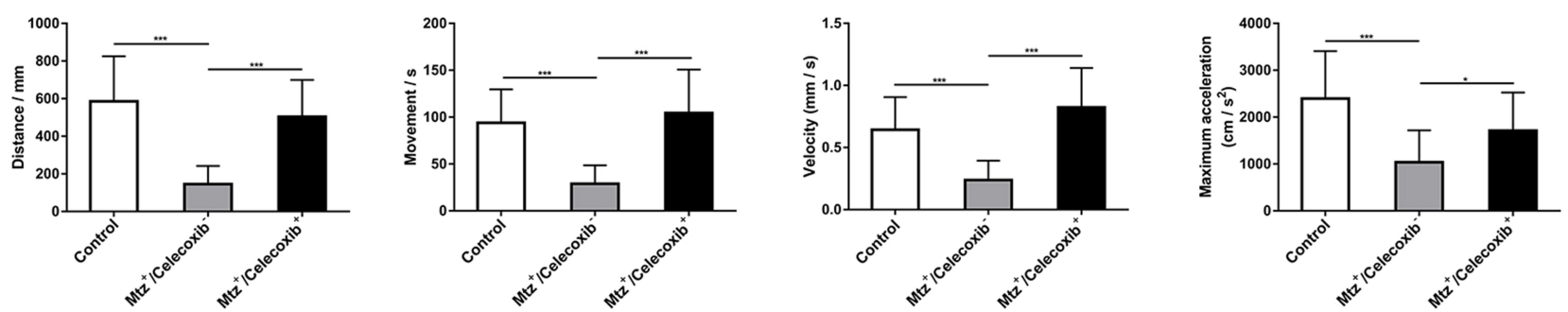

Figure 5 Analysis of the locomotor ability of demyelinated larvae following celecoxib treatment. (A) Digital tracks and corresponding heat maps of larvae from the control, $\mathrm{Mtz}^{+} /$celecoxib $^{-}$, and $\mathrm{Mtz}^{+} /$celecoxib $^{+}$groups. (B) Statistical analysis of total distance, movement time, velocity, and maximum acceleration (ANOVA). $* P<0.05$; $* * * P<0.00 \mathrm{I}$. Abbreviation: ns, not significant. 

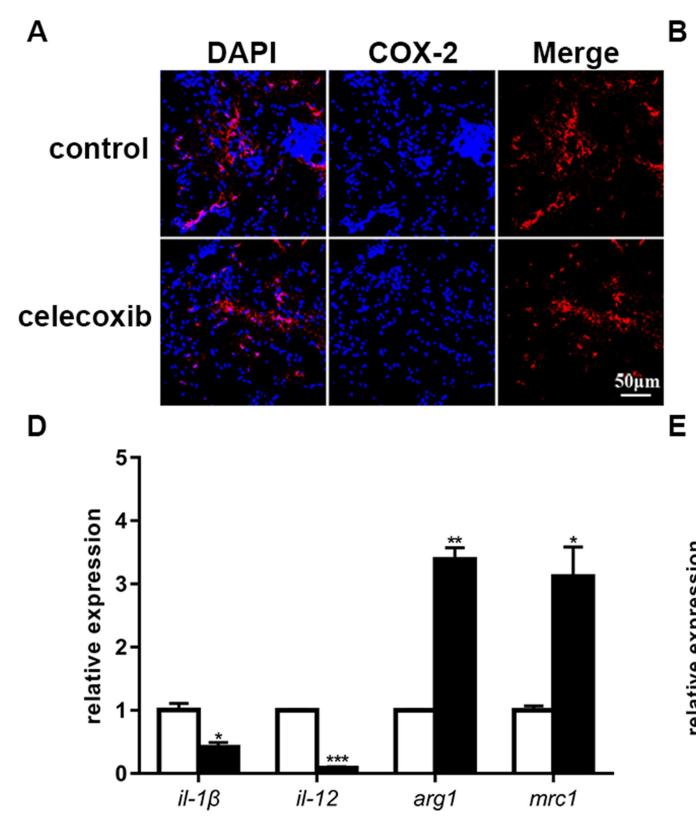

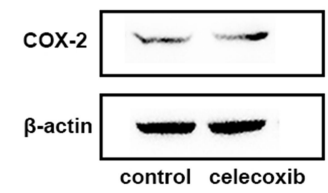

E

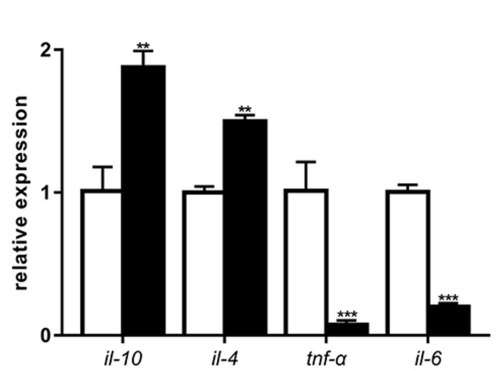

C

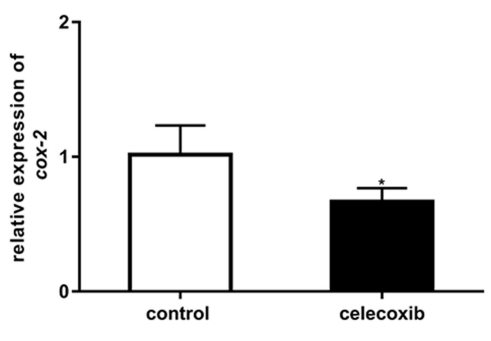

$\mathbf{F}$

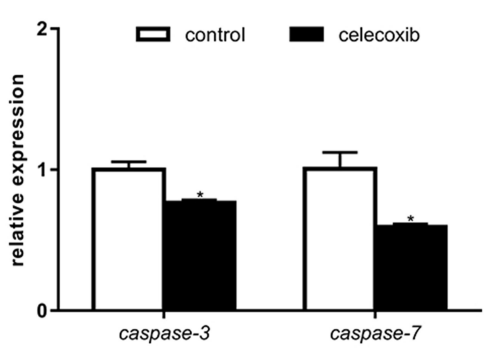

Figure 6 Celecoxib treatment inhibits COX-2 and alters the expression of microglia/macrophage markers, inflammatory cytokines and apoptotic factors. (A) Immunostaining for COX-2 in brain tissues from adult fish. (B) The expression of the COX-2 protein, as analyzed by Western blotting, at 6 dpt. (C) Relative cox-2 mRNA expression was decreased in the celecoxib-treated group compared to the control group (Student's $t$-test). (D) The relative expression of the microglia/macrophage markers il-I $\beta$, il-12, argl and $\mathrm{mrcl}$ in the control and celecoxib-treated groups (Student's $t$-test). (E) The relative expression of the inflammatory cytokines il- 10 , il-4, tnf- $\alpha$ and il-6 (Student's $t$-test). (F) The relative expression of caspase-3 and caspase-7 (Student's $t$-test). $* P<0.05 ; * * p<0.01 ; * * * p<0.001$.

inflammatory factors, and apoptotic factors in larvae following 3 days of celecoxib treatment and observed a similar tendency as in adults (Supplementary Figure $\underline{\text { S3A-D, }}$ Student's $t$-test, $\quad * P \quad<0.05, \quad * * P \quad<0.01$, $* * * P<0.001)$. Taken together, the above data reveal that celecoxib inhibits the expression of the neuroinflammatory factor COX-2, improves the immune and inflammatory microenvironments and suppresses apoptotic events.

\section{Discussion}

In this study, a stable and practical demyelination model was induced in Tg (mbp:nfsB-egfp) fish with Mtz. In adults, effective oligodendrocyte ablation was verified in situ and quantitatively. First, a remarkable decrease in the number of GFP-positive cells was observed in the brain, spinal cord, and optic nerve. Previously, we studied demyelination in the spinal cord using the same transgenic model. ${ }^{17}$ However, the overwhelming majority of patients who develop MS first present with a clinically isolated syndrome that involves the optic nerve, brainstem, or spinal cord. ${ }^{24}$ Therefore, we also explored pathological changes in the brain and optic nerve. Second, the expression of MBP protein and mRNA was also significantly decreased. It has been reported that a reduction in mbp gene transcription products or synthesis defects can result in delayed myelination. ${ }^{25}$ Our results also confirmed this finding. Because the larval zebrafish were relatively small in size, it was convenient to observe GFP expression in the spinal cord in whole mounts in real-time. The weakened and discontinuous fluorescence signals resulting from significantly reduced MBP protein expression verified the effective demyelination in larvae. To some extent, this primary demyelination in both adult and larval $\mathrm{Tg}$ (mbp: nfsB-egfp) fish mimics the early pathological events of $\mathrm{MS}^{26,27}$

It was important to evaluate the in vivo toxicity of celecoxib before we investigated its therapeutic effect. Following 72 hours of exposure, celecoxib had little effect on zebrafish development. The concentration of celecoxib treatment used in this study in both demyelinated adults and larvae was $2 \mu \mathrm{M}$. This dose is almost equivalent to the oral administration dose. The rate and effect of absorption of oral celecoxib is rapid and good, with $97 \%$ plasma protein binding, suggesting that it is extensively distributed in tissues. ${ }^{28}$ Because oligodendrocytes are specifically labeled with GFP in Tg (mbp:nfsB-egfp), we assessed the status of remyelination in adults following 6 days of celecoxib treatment through two approaches. First, GFPexpressing cells were quantified by cell sorting. Second, the in situ expression of GFP was examined by 
immunohistochemistry. The number of oligodendrocytes was significantly increased in the celecoxib-treated group compared to the control group. Moreover, the localization of GFP-positive cells (including in the brain, spinal cord, and optic nerve) was very similar to that observed before Mtz induction. We also treated demyelinated larvae with celecoxib for 3 days. The GFP signals in the spinal cord were increased in the celecoxib-treated group compared to the control group, and the continuous distribution was restored in the celecoxib-treated group. The expression of mbp was also upregulated. Moreover, we measured functional changes. In most MS patients, the clinical manifestations involve motor, sensory, visual, and autonomic nervous system dysfunction. ${ }^{29}$ Surprisingly, locomotor ability was dramatically restored to almost physiological levels in the celecoxib-treated group. Another noteworthy finding was the different treatment times between adults and larvae. We speculated that this was due to the agerelated decline in the regeneration rate. In animal models and MS patients, it was evident that the efficiency of CNS remyelination decreases with age. ${ }^{30}$ It has been reported that celecoxib has potential neuroprotective effects. In a rat model of hypoxia-reoxygenation, celecoxib is one of the most effective NSAIDs for preventing nerve cell death. ${ }^{31}$ In a middle cerebral artery occlusion model, celecoxib treatment can prevent or delay the occurrence of selective neuronal loss, inhibit glial activation, and improve neurological deficits. ${ }^{32}$ Our findings revealed that celecoxib can significantly increase the number of oligodendrocytes; hence, the morphology and function of the myelin sheath can be restored in a short period.

In MS patients, inflammation, the immune response, and apoptosis play pivotal roles in the nervous system. ${ }^{33,34}$ Microglia and macrophages mediate cell damage or neuroprotection and are involved in the pathogenesis of MS; at the early stage, microglia and macrophages are activated toward the classical proinflammatory M1 phenotype. As antigenpresenting cells, M1 microglia and macrophages also participate in activating helper T cells such as Th1 and Th17 cells to support immune responses. At later stages, the antiinflammatory M2 phenotype is predominant in the CNS. ${ }^{35}$ M2 microglia and macrophages can promote the differentiation of Th2 cells and regulatory $\mathrm{T}$ cells to inhibit Th1 cell activity and proinflammatory cytokine release. ${ }^{36,37}$ In response to distinct microenvironmental cues, a fully polarized M1 or M2 subpopulation can reverse its phenotype and function. In this study, we found decreased expression of M1 phenotype markers (il-1 $\beta$ and il-12) and increased expression of M2 phenotype markers (arg1 and mrc1). The different subtypes not only play a key role in the immune response and protect the organism against infection but also regulate inflammation. ${ }^{38}$ The upregulation of antiinflammatory cytokines (il-4 and il-10) and downregulation of pro-inflammatory mediators (tnf- $\alpha$ and il-6) further confirmed the transition of microglia and macrophages from the M1 to the M2 phenotype since pro-inflammatory mediators are released by M1 microglia and macrophages, ${ }^{39,40}$ while anti-inflammatory cytokines are released by M2 microglia and macrophages. ${ }^{41}$ The correlation between COX-2 inhibition and apoptosis was also addressed in this study. In a Theiler's murine encephalomyelitis virus (TMEV) model, the COX-2 protein is colocalized with the apoptotic mediator caspase-3. ${ }^{9}$ In a cuprizone-induced demyelination model, COX-2 activity is correlated with caspase-3 activation. ${ }^{42}$ Whether mediated by the extrinsic or intrinsic pathway, apoptosis depends on the activity of two endapoptotic effector enzymes, namely, caspase-3 and caspase-7. ${ }^{43}$ Following celecoxib treatment, the expression of caspase- 3 and caspase-7 was significantly decreased. Our findings reveal for the first time that celecoxib treatment can improve the inflammatory and immunity response and attenuate caspase-dependent apoptosis, leading to rapid tissue regeneration.

\section{Conclusion}

The main conclusions of this study are as follows: (1) celecoxib treatment promotes the regeneration of oligodendrocytes and contributes to the structural and functional recovery of the myelin sheath; (2) by selectively inhibiting COX-2, celecoxib can improve the immune and inflammatory microenvironments and suppress caspasedependent apoptosis, thus exerting a therapeutic effect against demyelination.

\section{Acknowledgments}

This work was supported by Chinese National Natural Science Foundation (No. 81671179 and 81971739), the National Key R\&D Program of China (No. 2017YFA0103200), and Municipal Student Innovation and Entrepreneurship Training Program (No. 202010055405).

\section{Disclosure}

The authors report no conflicts of interest in this work. 


\section{References}

1. Kim S, Lee YI, Chang KY, et al. Promotion of remyelination by sulfasalazine in a transgenic zebrafish model of demyelination. Mol Cells. 2015;38(11):1013-1021.

2. Bercury KK, Macklin WB. Dynamics and mechanisms of CNS myelination. Dev Cell. 2015;32(4):447-458. doi:10.1016/j.devcel.20 15.01.016

3. Ceci ML, Mardones-Krsulovic C, Sanchez M, Valdivia LE, Allende ML. Axon-Schwann cell interactions during peripheral nerve regeneration in zebrafish larvae. Neural Dev. 2014;9:22. doi:10.1186/1749-8104-9-22

4. Dendrou CA, Fugger L, Friese MA. Immunopathology of multiple sclerosis. Nat Rev Immunol. 2015;15(9):545-558. doi:10.1038/ nri3871

5. Bramow S, Frischer JM, Lassmann H, et al. Demyelination versus remyelination in progressive multiple sclerosis. Brain. 2010;133 (10):2983-2998. doi:10.1093/brain/awq250

6. Bodini B, Veronese M, Garcia-Lorenzo D, et al. Dynamic imaging of individual remyelination profiles in multiple sclerosis. Ann Neurol. 2016;79(5):726-738. doi:10.1002/ana.24620

7. Plemel JR, Liu WQ, Yong VW. Remyelination therapies: a new direction and challenge in multiple sclerosis. Nat Rev Drug Discov. 2017;16(9):617-634.

8. Pannunzio A, Coluccia M. Cyclooxygenase-1 (COX-1) and COX-1 inhibitors in cancer: A review of oncology and medicinal chemistry literature. Pharmaceuticals. 2018;11(4):101. doi:10.3390/ph11040 101

9. Carlson NG, Hill KE, Tsunoda I, Fujinami RS, Rose JW. The pathologic role for COX-2 in apoptotic oligodendrocytes in virus induced demyelinating disease: implications for multiple sclerosis. $J$ Neuroimmunol. 2006;174(1-2):21-31. doi:10.1016/j.jneuroim.20 06.01 .008

10. Kihara Y, Matsushita T, Kita Y, et al. Targeted lipidomics reveals mPGES-1-PGE2 as a therapeutic target for multiple sclerosis. Proc Natl Acad Sci U S A. 2009;106(51):21807-21812. doi:10.1073/ pnas.0906891106

11. Palumbo S, Toscano CD, Parente L, Weigert R, Bosetti F. Timedependent changes in the brain arachidonic acid cascade during cuprizone-induced demyelination and remyelination. Prostaglandins Leukot Essent Fatty Acids. 2011;85(1):29-35. doi:10.1016/j.plefa. 2011.04.001

12. Preston MA, Macklin WB. Zebrafish as a model to investigate CNS Myelination. Glia. 2015;63(2):177-193.

13. Emery B. Transcriptional and post-transcriptional control of CNS myelination. Curr Opin Neurobiol. 2010;20(5):601-607. doi:10.10 16/j.conb.2010.05.005

14. Preston MA, Finseth LT, Bourne JN, Macklin WB. A novel myelin protein zero transgenic zebrafish designed for rapid readout of in vivo myelination. Glia. 2019;67(4):650-667.

15. Fang YW, Lei XD, Li X, et al. A novel model of demyelination and remyelination in a GFP-transgenic zebrafish. Biol Open. 2015;4 (1):62-68. doi:10.1242/bio.201410736

16. Westerfield M The Zebrafish Book: A Guide for the Laboratory Use of Zebrafish (Brachydanio Rerio). Eugene OR: University of Oregon Press, Westerfield M; 1993.

17. Lei X, Cai S, Chen Y, et al. Down-regulation of interleukin 7 receptor (IL-7R) contributes to central nervous system demyelination. Oncotarget. 2017;8(17):28395-28407. doi:10.18632/oncotarget.16 081

18. Sun Y, Zhang G, He Z, Wang Y, Cui J, Li Y. Effects of copper oxide nanoparticles on developing zebrafish embryos and larvae. Int J Nanomedicine. 2016;11:905-918.

19. Huang T, Cui J, Li L, Hitchcock PF, Li Y. The role of microglia in the neurogenesis of zebrafish retina. Biochem Biophys Res Commun. 2012;421(2):214-220. doi:10.1016/j.bbrc.2012.03.139
20. Chung AY, Kim PS, Kim S, et al. Generation of demyelination models by targeted ablation of oligodendrocytes in the zebrafish CNS. Mol Cells. 2013;36(1):82-87. doi:10.1007/s10059-013-0087-9

21. Jung SH, Kim S, Chung AY, et al. Visualization of myelination in GFP-transgenic zebrafish. Dev Dyn. 2010;239(2):592-597. doi:10.10 02/dvdy.22166

22. Lyons DA, Naylor SG, Scholze A, Talbot WS. Kif1b is essential for mRNA localization in oligodendrocytes and development of myelinated axons. Nat Genet. 2009;41(7):854-858. doi:10.1038/ng.376

23. Miller RH. Regulation of oligodendrocyte development in the vertebrate CNS. Prog Neurobiol. 2002;67(6):451-467. doi:10.1016/ S0301-0082(02)00058-8

24. Thompson AJ, Baranzini SE, Geurts J, Hemmer B, Ciccarelli O. Multiple sclerosis. Lancet. 2018;391(10130):1622-1636. doi:10. 1016/S0140-6736(18)30481-1

25. Michel K, Zhao T, Karl M, Lewis K, Fyffe-Maricich SL. Translational control of myelin basic protein expression by ERK2 MAP kinase regulates timely remyelination in the adult brain. $J$ Neurosci. 2015;35 (20):7850-7865. doi:10.1523/JNEUROSCI.4380-14.2015

26. Lassmann H. Multiple sclerosis pathology. Cold Spring Harb Perspect Med. 2018;8(3):a028936. doi:10.1101/cshperspect.a028936

27. Mahad DH, Trapp BD, Lassmann H. Pathological mechanisms in progressive multiple sclerosis. T Lancet Neurol. 2015;14(2):183-193. doi:10.1016/S1474-4422(14)70256-X

28. van Midwoud PM, Sandker M, Hennink WE, de Leede LGJ, Chan A, Weinans $\mathrm{H}$. In vivo pharmacokinetics of celecoxib loaded endcapped PCLA-PEG-PCLA thermogels in rats after subcutaneous administration. Eur J Pharm Biopharm. 2018;131:170-177. doi:10. 1016/j.ejpb.2018.07.026

29. Yamout BI, Alroughani R. Multiple Sclerosis. Semin Neurol. 2018;38 (2):212-225. doi:10.1055/s-0038-1649502

30. Vaughn CB, Jakimovski D, Kavak KS, et al. Epidemiology and treatment of multiple sclerosis in elderly populations. Nat Rev Neurol. 2019;15(6):329-342. doi:10.1038/s41582-019-0183-3

31. Lopez-Villodres JA, De La Cruz JP, Munoz-Marin J, Guerrero A, Reyes JJ, Gonzalez-Correa JA. Cytoprotective effect of nonsteroidal antiinflammatory drugs in rat brain slices subjected to reoxygenation after oxygen-glucose deprivation. Eur J Pharm Sci. 2012;45 (5):624-631. doi:10.1016/j.ejps.2012.01.001

32. Santos-Galdiano M, Perez-Rodriguez D, Anuncibay-Soto B, et al. Celecoxib treatment improves neurologic deficit and reduces selective neuronal loss and glial response in rats after transient middle cerebral artery occlusion. J Pharmacol Exp Ther. 2018;367 (3):528-542. doi:10.1124/jpet.118.251264

33. Gurevich M, Achiron A. The switch between relapse and remission in multiple sclerosis: continuous inflammatory response balanced by Th1 suppression and neurotrophic factors. $J$ Neuroimmunol. 2012;252(1-2):83-88. doi:10.1016/j.jneuroim.2012.07.014

34. Macchi B, Marino-Merlo F, Nocentini U, et al. Role of inflammation and apoptosis in multiple sclerosis: comparative analysis between the periphery and the central nervous system. $J$ Neuroimmunol. 2015;287:80-87. doi:10.1016/j.jneuroim.2015.08.016

35. Hu X, Leak RK, Shi Y, et al. Microglial and macrophage polarization-new prospects for brain repair. Nat Rev Neurol. 2015;11(1):56-64. doi:10.1038/nrneurol.2014.207

36. Chu F, Shi M, Zheng C, et al. The roles of macrophages and microglia in multiple sclerosis and experimental autoimmune encephalomyelitis. J Neuroimmunol. 2018;318:1-7. doi:10.1016/j. jneuroim.2018.02.015

37. Tierney JB, Kharkrang M, La Flamme AC. Type II-activated macrophages suppress the development of experimental autoimmune encephalomyelitis. Immunol Cell Biol. 2009;87(3):235-240. doi:10.1038/icb.2008.99

38. Wynn TA, Chawla A, Pollard JW. Macrophage biology in development, homeostasis and disease. Nature. 2013;496(7446):445-455. doi:10.1038/nature12034 
39. Jiang HR, Milovanovic M, Allan D, et al. IL-33 attenuates EAE by suppressing IL-17 and IFN-gamma production and inducing alternatively activated macrophages. Eur J Immunol. 2012;42(7):18 04-1814. doi:10.1002/eji.201141947

40. Liu C, Li Y, Yu J, et al. Targeting the shift from M1 to M2 macrophages in experimental autoimmune encephalomyelitis mice treated with fasudil. PLoS One. 2013;8(2):e54841. doi:10.1371/journal. pone. 0054841

41. Laria A, Lurati A, Marrazza M, Mazzocchi D, Re KA, Scarpellini M. The macrophages in rheumatic diseases. J Inflamm Res. 2016;9:1-11.
42. Palumbo S, Toscano CD, Parente L, Weigert R, Bosetti F. The cyclooxygenase-2 pathway via the PGE (2) EP2 receptor contributes to oligodendrocytes apoptosis in cuprizone-induced demyelination. J Neurochem. 2012;121(3):418-427.

43. Green DR, Llambi F. Cell death signaling. Cold Spring Harb Perspect Biol. 2015;7(12):a006080. doi:10.1101/cshperspect.a006080

\section{Publish your work in this journal}

The Journal of Inflammation Research is an international, peerreviewed open-access journal that welcomes laboratory and clinical findings on the molecular basis, cell biology and pharmacology of inflammation including original research, reviews, symposium reports, hypothesis formation and commentaries on: acute/chronic inflammation; mediators of inflammation; cellular processes; molecular mechanisms; pharmacology and novel anti-inflammatory drugs; clinical conditions involving inflammation. The manuscript management system is completely online and includes a very quick and fair peerreview system. Visit http://www.dovepress.com/testimonials.php to read real quotes from published authors.

Submit your manuscript here: https://www.dovepress.com/journal-of-inflammation-research-journal 